\title{
Desenvolvimento e Validação da Escala de Crenças sobre a Doença Mental
}

\section{Development and Validation of the Beliefs about Mental Illness Scale}

\author{
Silvana Carneiro Maciel ${ }^{*},{ }$, Cícero Roberto Pereira ${ }^{b}$, Tiago Jessé Souza de Lima ${ }^{a}$ \\ \& Luana Elayne Cunha de $\operatorname{Souza}^{c}$ \\ ${ }^{a}$ Universidade Federal da Paraíba, João Pessoa, PB, Brasil, ${ }^{b}$ Universidade de Lisboa, Lisboa, Portugal \\ \& ${ }^{c}$ Universidade de Fortaleza, Fortaleza, CE, Brasil
}

\begin{abstract}
Resumo
Este artigo tem por objetivo desenvolver e validar a Escala de Crenças sobre a Doença Mental (ECDM), elaborada para avaliar as crenças subjacentes à natureza da doença mental. No Estudo 1 foram elaborados os itens da ECDM com base em 60 entrevistas com profissionais da saúde mental, familiares de doentes mentais hospitalizados e grupos focais de estudantes de Psicologia. O Estudo 2 foi realizado com 491 estudantes universitários, com média de idade de 23 anos $(D P=6)$, e teve por objetivo testar a validade fatorial e consistência interna da medida. O Estudo 3 foi realizado com 254 estudantes, com idade média de 25 anos $(D P=8)$, e buscou replicar o estudo anterior por meio de uma abordagem confirmatória. Ao longo de três estudos a ECDM demonstrou uma estrutura fatorial estável, constituída por seis fatores (psíquico, socioeconômico, biológico, uso de drogas, religioso e contingencial) com boa consistência interna. A versão final da escala ficou composta por 30 itens. Palavras-chave: Crenças, doença mental, escala, validação.
\end{abstract}

\begin{abstract}
The aim of this study is to develop and validate a new instrument, the Beliefs about Mental Illness Scale (BAMIS). This scale was designed to evaluate the beliefs underlying the nature of mental illness. In Study 1, items were developed based on 60 interviews with mental health professionals, relatives of mental inpatients, and focus groups with psychology students. Study 2 was conducted with 491 college students, at the mean age of 23 years $(S D=6.00)$, and aimed to test the validity and internal consistency of the scale. Study 3 was performed with 254 students at the mean age of 25 years $(S D=8.00)$ and aimed to replicate the previous study using a confirmatory approach. Across three studies, the BAMIS demonstrated a stable factor structure, consisting of six factors (psychological, socioeconomic, biological, drug use, religious and contingency), all with high internal consistency coefficients. The final version of the scale was composed of thirty items.

Keywords: Beliefs, mental health, scale, validation.
\end{abstract}

As pessoas portadoras de doença mental são recorrentemente alvos de preconceito, de estigmatização e das mais severas formas de exclusão social (Corrigan, Markowitz, Watson, Rowan, \& Kubiak, 2003; Maciel, Barros, Camino, $\&$ Melo, 2011). No Brasil e em outros países, diversos estudos têm sido desenvolvidos acerca do conceito de doença mental, das atitudes individuais e coletivas frente aos doentes mentais e também sobre os serviços de assistência

"Endereço para correspondência: Universidade Federal da Paraíba, Programa de Pós-Graduação em Psicologia Social, João Pessoa, PB Brasil 58051-900. Fone: (83) 8807-4781. E-mail: silcamaciel@gmail.com, cico.sec@ gmail.com, tiago.souzalima@hotmail.com e luana_elayne@hotmail.com

Este artigo foi financiado pela Coordenação de Aperfeiçoamento de Pessoal de Nível Superior (CAPES) por meio de uma bolsa concedida à primeira autora para a realização de estudos pós-doutorais no Instituto de Ciências Sociais da Universidade de Lisboa. oferecidos a estes doentes (Gonçalves, Abelha, Legay, \& Lovisi, 2008; Maciel, Barros, Silva, \& Camino, 2009; Osinaga, Furegato, \& Santos, 2004; Pasquali, Nogueira, Martins, \& Martins, 1987; Pedrão et al., 2005). No entanto, são poucos os estudos que têm por objetivo avaliar quais as crenças socialmente compartilhadas sobre as causas da doença mental e como estas crenças estruturam as atitudes e os comportamentos das pessoas em relação aos doentes mentais (Morant, 2006; Rodrigues \& Figueiredo, 2003; Tsu \& Tofolo, 1990).

De acordo com Lacerda, Pereira e Camino (2002), a análise dessas crenças é fundamental porque as representações sobre a natureza dos grupos sociais funcionam como princípios organizadores que motivam a expressão de preconceito e discriminação. Diversos estudos têm demonstrado empiricamente que as crenças sobre a natureza dos grupos motivam a expressão de preconceito e discriminação contra estes grupos (Pereira, Torres, Falcão, 
\& Pereira, 2013; Read, Haslam, Sayce, \& Davies, 2006; Schlier, Schmick, \& Lincoln, 2014). Identificar estas crenças se torna mais relevante no contexto da implantação da Reforma Psiquiátrica e dos serviços substitutivos de saúde mental que promovem o desenvolvimento de uma rede de assistência comunitária. Necessariamente, tais providências motivam a sociedade a encontrar mecanismos de integração dos doentes mentais à sua vida cotidiana.

Neste sentido, o presente trabalho tem como objetivo propor a Escala de Crenças Sobre a Doença Mental, que pode ser empregada para avaliar as crenças compartilhadas sobre a natureza da doença mental. Embora existam escalas acerca da doença mental disponíveis na literatura, elas não tratam especificamente das crenças sobre a sua etiologia, mas abordam outro aspecto relevante sobre este tema que são as atitudes frente à doença mental, tal qual a Community Attitudes Toward Mentally III (Gonçalves et al., 2008; Taylor \& Dear, 1981), a Escala de Atitudes Frente ao Doente (Pasquali et al., 1987), ou a escala de Opiniões sobre a Doença Mental (Pedrão et al., 2005). Já aquelas desenvolvidas para avaliar especificamente as crenças apresentam baixa consistência interna (Lincoln, Arens, Berger, \& Rief, 2008), ou faltam evidências de sua validade de construto e consistência interna (Link, Phelan, Bresnahan, Stueve, \& Pescosolido, 1999; Loureiro, Dias, \& Aragão, 2008). Neste sentido, optou-se por construir uma nova escala que abarcasse o conteúdo socialmente compartilhado acerca das causas da natureza da saúde mental e apresentasse parâmetros satisfatórios de validade e precisão.

\section{Crenças sobre a Etiologia da Doença Mental}

Nos dias atuais, as crenças sobre a etiologia da doença mental são bastante heterogêneas e carecem de consenso, o que permanece um desafio para a ciência psicológica (Morant, 2006). Embora muitas resistências tenham sido ultrapassadas para que a atribuição sobrenatural desse lugar a novas ideias, muitas das representações construídas nos séculos anteriores podem ainda se fazer presentes atualmente. $O$ fato de as representações serem social e historicamente construídas faz crescer a importância do resgate das concepções sobre a natureza do adoecer mental.

As primeiras ideias sobre saúde e doença invocavam fatores sobrenaturais. A doença mental era vista como resultado da ação de entidades externas, como demônios, espíritos e divindades (Pessotti, 2001). Foi Hipócrates quem estabeleceu uma passagem do sobrenatural para o natural. Contudo, essa ênfase em aspectos naturais perdeu relevância durante a Idade Média, período em que prevaleceram as explicações que invocavam motivações demoníacas e de ordem religiosa para a ocorrência das doenças mentais. Com o rompimento do sistema feudal, a loucura passou a ser representada não apenas como um problema de natureza religiosa, mas também como uma categoria socialmente problemática, motivando a criação dos hospitais psiquiátricos. O modelo biomédico se instalou como paradigma norteador, adotando um modelo organicista (Wetzel, Kantorski, Olschowsky, Schneider, \& Camatta, 2011). Neste sentido, as representações sobre a doença mental incorporaram fatores de natureza biológica. Posteriormente, Freud (1895/1996) propôs uma nova concepção da doença mental, dando menos importância a fatores orgânicos e propondo, como causas, fatores relacionados com a subjetividade do sujeito, com destaque para os conflitos motivacionais que estão na base das tensões entre a sexualidade e as normas culturais.

Historicamente observamos a construção de três grandes explicações sobre as causas da doença mental, uma ligada a crenças de natureza religiosa ou sobrenatural, outra orgânica ou biológica e por fim, a psicológica. Esse resgate é necessário a fim de se obter uma melhor compreensão de como estas crenças estão colocadas na atualidade (Oliveira, Lima, Silva, Oliveira, \& Alves, 2011). Diversos estudos têm demonstrado que as pessoas recorrem a estas e outras crenças, em maior ou menor grau, para explicar diferentes doenças mentais (Haqanee, Lou, \& Lalonde, 2014; Link et al., 1999; Minayo, 1988; Schlier et al., 2014).

Por exemplo, Minayo (1988) fez entrevistas com famílias de doentes mentais sobre a concepção que elas tinham acerca da etiologia da doença mental, e observou que as explicações se organizavam em quatro níveis: natural, psicossocial, socioeconômico e sobrenatural. Loureiro et al. (2008), em um estudo sobre as crenças e atitudes acerca das doenças mentais realizado em Portugal, demonstraram que os participantes atribuíam a doença mental a traumas emocionais e profissionais $(77,44 \%)$, a causas associadas a deterioração física e envelhecimento $(59,75 \%)$ e a causas associadas a traumas à nascença, doença, e comportamentos de risco (38,31\%). Indo mais além, Link et al. (1999) demonstraram que a atribuição causal varia de acordo com a doença mental em questão. Estes autores solicitaram aos participantes que avaliassem o quanto uma doença mental tem como causa as seguintes opções: falta de caráter, desequilíbrio químico no cérebro, a forma como a pessoa foi criada, circunstanciais estressantes, problemas genéticos, e a vontade de Deus. Para a esquizofrenia, dentre as causa mais aceitas estavam as circunstâncias estressantes (90\%), seguidas pelo desequilíbrio químico $(84,6 \%)$, problemas genéticos $(67 \%)$, a forma como a pessoa foi criada $(45 \%)$, falta de caráter (32,8\%), e vontade de Deus (17\%).

De acordo com Angermeyer, Holzinger, Carta e Schomerus (2011), estudos realizados nos EUA, Austrália e Alemanha mostraram um aumento global nas atribuições causais biogenéticas dos transtornos mentais nas duas últimas décadas. Verificou-se que cada vez mais as doenças mentais, como a depressão e a esquizofrenia, são atribuídas a doença cerebral, desequilíbrios químicos no cérebro ou causas genéticas. Com relação as crenças causais mágico-religiosas acerca da doença mental, são poucos os estudos que abordam essas crenças. Em estudo realizado na Nigéria (Gureje, Olley, Oluwanuga, \& Kola, 2006), verificou-se que, de uma amostra de 1163 pessoas da população geral, $84,6 \%$ possuíam crenças causais exclusivamente biopsicossociais, enquanto que $15,4 \%$ ti- 
Maciel, S. C., Pereira, C. R., Lima, T. J. S. \& Souza, L. E. C. (2015). Desenvolvimento e Validação da Escala de Crenças sobre a Doença Mental.

nham crenças exclusivamente mágico-religiosas a respeito das causas da doença mental. Constatou-se que ambos os grupos possuíam um fraco conhecimento acerca das doenças mentais e atitudes predominantemente negativas, tendo o grupo das crenças biopsicossociais uma atitude mais tolerante e menos estigmatizante do que o grupo das crenças mágico-religiosas.

Alguns estudos têm demonstrado que a informação sobre a etiologia da doença mental está associada a uma atitude mais positiva ou negativa, a depender da crença em questão (Haslam, 2011; Read et al., 2006). Até recentemente uma abordagem objetivava reduzir o estigma da doença mental enfatizando causas biológicas e genéticas, ou seja, de que a doença mental era uma doença como qualquer outra (Phelan, 2002). Outros estudos também têm demonstrado que as causas psicossociais podem contribuir para a redução do estigma (Lincoln et al., 2008). As crenças também podem aumentar as atitudes negativas, tais como necessidade de manter distância e percepção de periculosidade (Pescosolido et al., 2010). Todavia, estes achados têm apresentado grande variabilidade e inconsistência entre os estudos (Read et al., 2006; Schlier et al., 2014). Ademais, pouca atenção tem sido dada à análise do papel de outras crenças para a compreensão da etiologia da doença mental.

Apesar da relevância do tema, existem poucas escalas que têm por objetivo avaliar as crenças sobre as causas da doença mental. Em um levantamento feito no Google Acadêmico (20 de outubro de 2013), com as palavras-chave "escala crenças doença mental" e "escala causas doença mental", foi encontrada apenas uma escala específica para avaliar as crenças, o Questionário de causas acerca da doença mental (Loureiro et al., 2008). Todavia, o artigo em questão não apresenta evidências de validade e consistência interna. Em outra pesquisa no Google Acadêmico (20 de outubro de 2013), com as palavras-chave "beliefs mental illness scale" e "causes mental illness scale", foram encontradas duas escalas que também apresentam problemas quanto aos seus parâmetros psicométricos. A Preexisting causal beliefs (Lincoln et al., 2008; Schlier et al., 2014) apresentou baixa consistência interna para dois dos três fatores (crenças biológicas, $\alpha$ $=0,56$; outras crenças, $\alpha=0,30$ ). Já a escala empregada por Link et al. (1999) consistia apenas de um item para cada crença proposta, não tendo sido reportados seus parâmetros psicométricos.

O papel das crenças sobre as causas da doença mental é relevante na medida em que os indivíduos as utilizam para embasar suas atitudes e comportamentos frente aos doentes mentais (Oliveira et al., 2011; Read et al., 2006; Schlier et al., 2014). Neste sentido, a escala proposta neste trabalho pode contribuir para o estudo dos fatores psicossociais que motivam não apenas o preconceito, mas também o apoio que as pessoas dão às políticas sociais que promovem a inclusão dos doentes mentais. De fato, o instrumento proposto vem preencher uma lacuna, no Brasil, de medidas sobre as crenças das causas da doença mental. A escala foi construída em três estudos. No primeiro estudo foi feito um levantamento e sistematização dos tipos de crenças socialmente compartilhadas acerca das causas da doença mental. Com base nessa sistematização, o segundo e terceiro estudo apresentam a construção da escala e evidências empíricas de sua validade de construto e consistência interna.

\section{Estudo 1 - Crenças Socialmente Compartilhadas Acerca das Causas da Doença Mental}

O Estudo 1 teve por objetivo fazer um levantamento e sistematização dos tipos de crenças socialmente compartilhadas acerca das causas da doença mental a partir da análise de conteúdo de entrevistas realizadas com participantes de contextos diversos.

\section{Participantes}

Foram entrevistadas 60 pessoas, sendo 25 profissionais da saúde mental, 25 familiares de doentes mentais institucionalizados e 10 estudantes universitários.

\section{Instrumentos}

Foram realizadas entrevistas semiestruturadas, individuais, com uso do gravador, garantindo-se o anonimato dos participantes. A entrevista consistia em uma questão na qual deveriam elencar, livremente, os motivos que ele acredita que levariam uma pessoa a se tornar doente mental.

\section{Procedimentos e Análise dos Dados}

As entrevistas foram realizadas em sala apropriada, de acordo com a disponibilidade dos participantes. $\mathrm{O}$ anonimato das respostas foi garantido, sendo que os participantes tiveram que assinar uma autorização prévia, atendendo aos critérios éticos em vigor. As entrevistas foram transcritas e analisadas seguindo a técnica de Análise de Conteúdo Temática proposta por Bardin (1977) com categorias pós-definidas.

\section{Resultados e Discussão}

Uma análise qualitativa das respostas revelou conteúdos que puderam ser agrupados em seis conjuntos de crenças que explicam a doença mental. Foram elencadas tanto explicações científicas, como as do senso comum. Os dados foram agrupados nas seguintes categorias:

1. Explicações religiosas: "são tentações malignas do mundo que pegam nas pessoas fracas"; "é o inimigo que induz, se coloca no pensamento da pessoa... ouve voz, aquilo ali é coisa do Satanás".

2. Explicações biológicas: "parece que já vem de familia porque os meus filhos o mais velho e o caçula já estão adoecendo também"; "causas hereditárias que são estas que por si só eclodem devido a carga genética".

3. Explicações psicológicas: “. . . acho que deve ser preocupação"; "pressão psicológica, muita pressão", "pessoas sentimentais... trauma de criança". 
4. Explicações de natureza socioeconômica, como a importância das condições de pobreza: "um motivo, lá em casa nunca teve boa alimentação"; "má alimentação".

5. Explicações pautadas no consumo de drogas ou bebidas alcoólicas: "... tomar anabolizante, achamos que foi isto que atingiu os nervos dele"; "consequências do uso abusivo de álcool e de drogas ilícitas que levam a deterioração do cérebro".

6. Explicações pautadas em fatores contingenciais: " $a$ doença mental é devida a pancadas na cabeça"; "queda que a pessoa cai e machuca a cabeça".

Os resultados aqui obtidos encontram respaldo em outros estudos, tal como o realizado por Minayo (1988) e por Ngokwey (1988) que, adotando uma metodologia parecida, encontraram quatro conjuntos de crenças: natural, psicossocial, socioeconômica e sobrenatural. As frases evocadas pelos participantes foram utilizadas como base para a construção de um banco de itens que foram posteriormente empregados para construir a Escala de Crenças sobre a Doença Mental (ECDM), como descrito no Estudo 2.

\section{Estudo 2 - Construção da ECDM}

O Estudo 2 teve por objetivo relatar a construção da ECDM, com base nos itens elaborados a partir das frases extraídas das entrevistas do Estudo 1, e testar seus parâmetros de validade e precisão. Para se obter a versão final da escala, foi realizada uma análise de juízes e posteriormente uma validação semântica. Por fim, foram analisadas a validade de fatorial e consistência interna do instrumento.

\section{Participantes}

Participaram deste estudo 491 estudantes universitários, com média de idade de 23 anos $(D P=6)$, sendo a maioria do sexo feminino $(75,2 \%)$, distribuídos entre os cursos de enfermagem, medicina, psicologia, serviço social e terapia ocupacional. Quanto ao contato com doentes mentais, 23,6\% declararam ter um doente mental na família.

\section{Instrumento}

A versão preliminar da ECDM contou com 37 itens, construídos a partir das entrevistas do Estudo 1. Os itens foram elaborados de modo a representar cinco possíveis conjuntos de crenças, escolhidos com base na literatura consultada e nas entrevistas, a saber: psicológico, biológico, religioso, socioeconômico e contingenciais (uso de drogas e acidentes/traumatismos cranianos). As três primeiras crenças são comumente descritas na literatura (Lincoln et al., 2008; Link et al, 1999; Minayo, 1988; Schlier et al., 2014), e as duas últimas foram incluídas com base nos conteúdos extraídos das entrevistas.

Os itens elaborados foram submetidos à análise de sete juízes, mestre e doutores no tema. Adotou-se um critério mínimo de $70 \%$ de concordância entre os juízes para verificar a validade aparente e a pertinência de cada item a sua respetiva categoria. Foram excluídos 03 itens que não alcançaram o critério estabelecido. Após as análises de juízes, a primeira versão da ECDM ficou composta por 34 itens. Esta versão foi submetida a uma validação semântica com um grupo de cinco participantes, com diferentes níveis de formação, para verificar a compreensão dos itens e formato da escala de resposta. Alguns exemplos de itens são os seguintes: "A doença mental pode ser causada por uma decepção amorosa" (psicológico); "Pancadas na cabeça causam doença mental” (contingencial); "A doença mental ocorre por vontade de Deus" (religioso); "A pobreza é um dos determinantes da doença mental" (socioeconômico); "O doente mental já nasce com a doença" (biológico). A instrução de resposta adotada solicita que o participante indique o quanto concorda com cada uma das afirmações, utilizando uma escala do tipo Likert que varia de Discordo totalmente (1) a Concordo totalmente (5).

\section{Procedimento}

O instrumento foi aplicado em sala de aula, tendo sido respondido individualmente. Todos os sujeitos foram informados do caráter voluntário da sua participação na pesquisa, da garantia de anonimato das respostas dadas e do respeito às diretrizes éticas que regem a pesquisa com seres humanos. O projeto foi aprovado pelo Conselho de Ética em Pesquisa da Universidade Federal da Paraíba (protocolo $\mathrm{n}^{\circ}$ 138515/outubro 2012), obedecendo a todos os cuidados de pesquisa, de acordo com a Resolução 466/2012.

\section{Análise dos Dados}

Foram realizadas análises exploratórias e de consistência interna da medida. O método de extração adotado foi o dos Componentes Principais (ACP). Como critérios para a extração dos componentes, adotou-se o valor próprio igual ou maior que 1 (critério de Kaiser), a análise paralela de Horn (1965) e a interpretabilidade dos componentes extraídos (Garson, 2012). O método de rotação utilizado foi o varimax. Foi adotado como ponto de corte para a carga fatorial o valor de 0,40 (Matsunaga, 2010). No caso de um item obter cargas fatoriais elevadas em um ou mais componentes, optou-se por reter o item no componente no qual apresentou maior carga. Para avaliar a consistência interna da medida, foram utilizados o alfa de Cronbach, considerando-se como adequados os valores acima de 0,70 (Nunnally, 1991), e o índice de homogeneidade (correlação média interitens), assumindo como adequados os valores iguais ou superiores a 0,20 (Clark \& Watson, 1995).

\section{Resultados}

$\mathrm{O} \mathrm{KMO}=0,88$ e o teste de esfericidade de Bartlett, $\chi^{2}(561)=6029,3, p<0,001$, foram considerados satisfatórios, o que permitiu prosseguir com as análises. $\mathrm{Na}$ primeira ACP, sem determinar o número de componentes a extrair, foram obtidos 8 fatores com valores próprios maiores do que 1. A análise paralela de Horn (1965) indicou a extração de cinco fatores com valores próprios acima daqueles obtidos randomicamente. Como a solução com oito fatores carece de interpretabilidade, optou-se por realizar uma nova análise fixando a extração de cinco 
Maciel, S. C., Pereira, C. R., Lima, T. J. S. \& Souza, L. E. C. (2015). Desenvolvimento e Validação da Escala de Crenças sobre a Doença Mental.

componentes, conforme a proposta inicial e a indicação da análise paralela. Os resultados da nova ACP apontam que os itens dos fatores "psicológico" e "socioeconômico" saturaram em um mesmo componente, e o componente "contingencial" dividiu-se em dois, um com os itens relacionados ao consumo/abuso de drogas e o outro com a causa de traumatismo craniano, os outros componentes extraídos foram o religioso e biológico. O percentual de variância explicada foi de 50,01\% para os cinco fatores.

Levando-se em conta, que os itens de crenças distintas saturaram em um mesmo componente (como é o caso das crenças psicológica e socioeconômica), e a emergência dos itens de consumo/abuso de drogas separados das crenças contingenciais (traumatismo craniano), optou-se por proceder nova ACP definindo a extração de seis fatores. Esta solução hexafatorial é compatível com as categorias obtidas no Estudo 1. A solução final reteve 24 itens, sendo excluídos sete itens por apresentarem carga fatorial abaixo de 0,40 . Os seis fatores explicaram $58,6 \%$ da variância. Os itens, as cargas fatoriais e os indicadores de consistência interna são apresentados na Tabela 1 .

O componente 1, denominado de crenças Psicológicas $\left(\alpha=0,80, r_{\mathrm{m} . \mathrm{i}}=0,45\right)$, reuniu 5 itens, que falam sobre a natureza psicológica da doença mental. Este componente engloba problemas emocionais, de estresse e também traumas ocorridos na infância. O componente 2, denominado de crenças Religiosas $\left(\alpha=0,79, r_{\mathrm{m} . \mathrm{i}}=0,44\right)$, reuniu 5 itens e agrupa as explicações sobre uma suposta natureza religiosa ou sobrenatural da doença mental. Estas estão vinculadas à vontade de entidades religiosas que fogem ao controle individual, mas também envolvem a falta de fé e a fraqueza do indivíduo. O componente 3 , denominado de crenças Biológicas $\left(\alpha=0,75, r_{\text {m.i }}=0,37\right)$, reuniu 5 itens, que falam sobre a natureza orgânica da doença mental, envolvendo aspectos biológicos, genéticos e de desequilíbrio químico no cérebro. O componente 4 , denominado de Uso de Drogas $\left(\alpha=0,71, r_{\mathrm{m} . \mathrm{i}}=0,46\right)$, reuniu 3 itens com as crenças de que a doença mental surge a partir do abuso ou consumo excessivo de drogas, tanto de álcool como de outras drogas ilícitas. O componente 5 , denominado de crenças Socioeconômicas $\left(\alpha=0,65, r_{\mathrm{m} . \mathrm{i}}=0,39\right)$, reuniu 3 itens assentes nas crenças de que a doença mental surge a partir de questões de ordem econômica, como a pobreza, o desemprego e a falta de uma alimentação que supra as necessidades mínimas. O componente 6 , denominado crenças Contingenciais $\left(\alpha=0,55, r_{\mathrm{m} . \mathrm{i}}=0,29\right)$, reuniu 3 itens que apontam, os acidentes, os traumatismos cranianos e o estudo em excesso como as causas da doença mental; ou seja, trata-se de causas externas e/ou acidentais.

\section{Discussão}

O objetivo deste estudo foi construir uma Escala de Crenças sobre a Doença Mental (ECDM) e reunir evidências de validade fatorial e consistência interna deste instrumento. A escala ficou composta de 24 itens, distribuídos em seis tipos de crenças. A emergência das crenças psicológica, religiosa e biológica corroboram os achados de outros estudos descritos na literatura, nos quais estes três tipos de crenças são comumente relatados (Lincoln et al., 2008; Link et al, 1999; Schlier et al., 2014). Uma importante contribuição dos resultados aqui obtidos é a emergência de outros três conjuntos de crenças, a socioeconômica, de uso de drogas e contingenciais. Um estudo realizado por Minayo (1988) também demonstrou a emergência de explicações socioeconômicas para a doença mental e a crença no abuso de drogas também é identificada em outros estudos, mas como um tipo de perturbação mental e não como uma causa (Link et al., 1999). O instrumento apresenta evidências de validade fatorial e bons indicadores de consistência interna para a maior parte dos componentes (Clark \& Watson, 1995; Nunnally, 1991. Embora os componentes 5 e 6 tenham apresentado valores limítrofes de alfa de Cronbach, os índices de homogeneidade estão acima do recomendado. Tendo em conta esta limitação, um segundo estudo foi realizado com o objetivo de testar, em uma amostra diferente, a replicabilidade da estrutura fatorial aqui proposta.

\section{Estudo 3 - Análise Confirmatória da ECDM}

O objetivo deste estudo foi reunir evidência empírica adicional de validade fatorial e precisão da ECDM em uma nova amostra. Procurou-se testar, por meio de análise fatorial confirmatória, a replicabilidade da estrutura hexafatorial obtida no Estudo 1 e compará-la com soluções fatoriais alternativas. Inicialmente, foram elaborados mais seis itens (dois itens para as crenças de uso de drogas, socioeconômicas, e contingenciais), com o objetivo de equiparar o número de itens por fator (cinco para cada fator) e melhorar a consistência interna da escala.

\section{Participantes}

Participaram deste estudo 254 estudantes universitários, com média de idade de 25 anos $(D P=8,00)$, sendo a maioria do sexo feminino $(74,0 \%)$, distribuídos entre os cursos de enfermagem, medicina, psicologia e serviço social. Quanto ao contato com doentes mentais, 20,5\% declararam ter um doente mental na família.

\section{Instrumento}

A versão final da ECDM foi composta por 30 itens. Destes, 24 corresponderam à seleção descrita no Estudo 1 e outros seis itens foram elaborados neste estudo, de maneira que cada fator ficou representado por 5 itens. A elaboração destes itens passou pelo mesmo processo de análise de juízes, no qual os itens alcançaram $70 \%$ ou mais de concordância entre os juízes de que retratavam a crença para o qual tinham sido elaborados. Os juízes foram os mesmos que colaboraram no Estudo 2.

\section{Procedimentos}

$\mathrm{Na}$ coleta de dados, foram seguidos os mesmos procedimentos descritos no Estudo 1. 
Tabela 1

Cargas Fatoriais Após Rotação e Matriz de Correlações dos Componentes

\begin{tabular}{|c|c|c|c|c|c|c|}
\hline Itens resumidos & $\mathrm{C} 1$ & $\mathrm{C} 2$ & $\mathrm{C} 3$ & $\mathrm{C} 4$ & $\mathrm{C} 5$ & C6 \\
\hline 33. Excesso de tristeza & 0,75 & 0,19 & $-0,03$ & 0,18 & 0,12 & 0,12 \\
\hline 18. Instabilidade emocional & 0,75 & 0,04 & 0,12 & 0,06 & 0,02 & 0,11 \\
\hline 11. Problemas emocionais & 0,72 & 0,05 & 0,12 & 0,10 & 0,18 & $-0,07$ \\
\hline 20. Excesso de preocupação com a vida & 0,66 & 0,19 & $-0,12$ & 0,19 & 0,18 & 0,13 \\
\hline 13. Traumas psicológicos na infância & $\mathbf{0 , 6 3}$ & 0,00 & 0,25 & 0,14 & 0,10 & 0,00 \\
\hline 30. Espírito que entra na mente da pessoa & 0,04 & $\mathbf{0 , 8 7}$ & $-0,01$ & 0,10 & 0,05 & 0,03 \\
\hline 29. Fraqueza para resistir aos espíritos & 0,03 & $\mathbf{0 , 8 5}$ & $-0,03$ & 0,07 & 0,06 & 0,02 \\
\hline 15. Manifestações dos espíritos & 0,04 & $\mathbf{0 , 8 3}$ & 0,03 & 0,17 & 0,07 & $-0,05$ \\
\hline 25.Vontade de Deus & 0,10 & 0,56 & 0,02 & $-0,24$ & $-0,04$ & 0,04 \\
\hline 02. Falta de fé em Deus & 0,19 & $\mathbf{0 , 5 4}$ & $-0,06$ & $-0,10$ & 0,14 & 0,17 \\
\hline 17. Problemas biológicos & 0,08 & $-0,01$ & 0,77 & 0,00 & 0,08 & 0,03 \\
\hline 31. Distúrbio orgânico & $-0,08$ & 0,01 & $\mathbf{0 , 7 3}$ & 0,09 & 0,17 & $-0,05$ \\
\hline 12. Problemas orgânicos no cérebro & $-0,02$ & $-0,13$ & 0,71 & 0,09 & 0,08 & 0,08 \\
\hline 24. Fatores genéticos & 0,15 & 0,05 & 0,63 & 0,07 & $-0,04$ & 0,00 \\
\hline 16. Distúrbios cerebrais & 0,09 & 0,02 & 0,61 & 0,13 & $-0,13$ & 0,11 \\
\hline 22. Bebidas alcoólicas em demasia & 0,15 & 0,04 & 0,02 & $\mathbf{0 , 7 9}$ & 0,15 & 0,15 \\
\hline 27. Uso de drogas & 0,21 & $-0,04$ & 0,22 & $\mathbf{0 , 7 7}$ & $-0,02$ & 0,02 \\
\hline 32. Relacionada com o uso de drogas & 0,35 & 0,04 & 0,29 & $\mathbf{0 , 5 9}$ & 0,14 & 0,02 \\
\hline 08. A falta de alimentação quando criança & 0,07 & 0,10 & 0,20 & 0,07 & $\mathbf{0 , 7 8}$ & 0,08 \\
\hline 10. A pobreza & 0,27 & 0,13 & 0,00 & 0,07 & 0,68 & 0,12 \\
\hline 07. Desemprego & 0,48 & $-0,01$ & $-0,10$ & 0,12 & 0,62 & 0,04 \\
\hline 21. Pancadas na cabeça & 0,09 & 0,08 & 0,13 & 0,16 & $-0,03$ & $\mathbf{0 , 8 1}$ \\
\hline 01.Traumatismo craniano & $-0,04$ & 0,01 & 0,13 & 0,04 & 0,22 & $\mathbf{0 , 8 0}$ \\
\hline \multirow[t]{3}{*}{ 04. Estudar muito } & 0,34 & 0,09 & $-0,10$ & $-0,03$ & 0,04 & 0,44 \\
\hline & $0,23 *$ & & & & & \\
\hline & $0,15^{*}$ & $-0,02$ & & & & \\
\hline \multirow[t]{3}{*}{ Correlações } & $0,45^{*}$ & 0,07 & $0,31^{*}$ & & & \\
\hline & $0,27 *$ & $0,14^{*}$ & $0,14^{*}$ & $0,24 *$ & & \\
\hline & $0,49^{*}$ & $0,18^{*}$ & $0,14^{*}$ & $0,32 *$ & $0,27 *$ & - \\
\hline
\end{tabular}

Nota. Os valores em negrito indicam os itens retidos para cada componente.

Análise dos Dados

O pacote estatístico AMOS 18.0 foi empregado para realizar análises fatoriais confirmatórias, tendo em conta a matriz de covariância e adotando o estimador ML (Máxima Verossimilhança). Os casos omissos (missing data) compõem apenas $0,8 \%$ das observações e por esta razão foram substituídos pela média das respostas obtidas em cada item. Para conhecer o ajuste do modelo proposto e compará-lo com modelos alternativos, utilizaram-se os seguintes indicadores: a razão do qui-quadrado pelos graus 
Maciel, S. C., Pereira, C. R., Lima, T. J. S. \& Souza, L. E. C. (2015). Desenvolvimento e Validação da Escala de Crenças sobre a Doença Mental.

de liberdade $\left(\chi^{2} / g l\right)$, onde valores menores do que 5 indicam um ajuste adequado do modelo; o goodness of fit index (GFI), o adjusted goodness of fit index (AGFI) e o comparative fit index (CFI), onde valores iguais ou superiores a 0,90 indicam ajuste bom; e o root mean square error of approximation (RMSEA), cujos valores de 0,06 ou menor indicam ajuste satisfatório, aceitando-se aqueles de até 0,10 (Garson, 2012). Além destes indicadores, a diferença de qui-quadrado $\left(\Delta \chi^{2}\right)$ e o expected cross-validation index (ECVI) foram empregados para avaliar melhoras entre os modelos concorrentes testados. Valores significativos de $\Delta \chi^{2}$ e valores mais baixos de ECVI refletem um modelo com melhor ajuste (Garson, 2012).

Tabela 2

Índices de Ajuste dos Modelos Testados

\section{Resultados}

Inicialmente, realizou-se uma análise fatorial confirmatória, testando o modelo com os seis fatores proposto nos Estudos $1 \mathrm{e} 2$. Os indicadores de ajuste para este modelo podem ser observados na Tabela 2. O modelo proposto, denominado de modelo 1 , com seis fatores correlacionados, foi comparado com cinco modelos alternativos: modelo 2, seis fatores não correlacionados; modelo 3 , seis fatores preditos por um fator geral de segunda ordem; modelo 4 , cinco fatores correlacionados, com as crenças psicológicas e socioeconômicas agrupadas; modelo 5 , seis fatores com um fator de segunda ordem predizendo os fatores psicológico e socioeconômico; e modelo 6 , unifatorial de primeira ordem. Os índices de ajuste para os seis modelos testados são apresentados na Tabela 2.

\begin{tabular}{lcccccccc}
\hline Modelos & $\begin{array}{c}\chi^{2} \\
(g l)\end{array}$ & $\chi^{2} / g l$ & GFI & AGFI & CFI & $\begin{array}{c}\text { RMSEA } \\
(\text { IC }-90 \%)\end{array}$ & ECVI & $\begin{array}{c}\Delta \chi^{2} \\
(\Delta g l)\end{array}$ \\
\hline Modelo 1 & $\begin{array}{c}798,71 \\
(390)\end{array}$ & 2,05 & 0,82 & 0,79 & 0,86 & $\begin{array}{c}0,064 \\
(0,058-0,071)\end{array}$ & 3,75 & - \\
Modelo 2 & $\begin{array}{c}1171,28 \\
(405)\end{array}$ & 2,89 & 0,75 & 0,71 & 0,74 & $\begin{array}{c}0,086 \\
(0,081-0,092)\end{array}$ & 5,10 & $\begin{array}{c}372,57^{*} \\
(15)\end{array}$ \\
Modelo3 & $\begin{array}{c}823,44 \\
(399)\end{array}$ & 2,06 & 0,82 & 0,79 & 0,86 & $\begin{array}{c}0,065 \\
(0,059-0,071)\end{array}$ & 3,78 & $\begin{array}{c}24,73^{*} \\
(9)\end{array}$ \\
Modelo 4 & $\begin{array}{c}816,78 \\
(395)\end{array}$ & 2,07 & 0,82 & 0,79 & 0,86 & $\begin{array}{c}0,065 \\
(0,059-0,071)\end{array}$ & 3,78 & $\begin{array}{c}18,07^{*} \\
(5)\end{array}$ \\
Modelo 5 & $\begin{array}{c}803,15 \\
(393)\end{array}$ & 2,04 & 0,82 & 0,79 & 0,86 & $\begin{array}{c}0,064 \\
(0,058-0,071)\end{array}$ & 3,74 & $\begin{array}{c}4,44 \\
(3)\end{array}$ \\
Modelo 6 & $\begin{array}{c}1895,15 \\
(405)\end{array}$ & 4,68 & 0,61 & 0,55 & 0,49 & $\begin{array}{c}0,121 \\
(0,115-0,126)\end{array}$ & 7,96 & $\begin{array}{c}1096,44^{*} \\
(15)\end{array}$ \\
\hline
\end{tabular}

Nota. Modelo 1 = seis fatores correlacionados; Modelo 2 = seis fatores não correlacionados; Modelo 3 = seis fatores sendo preditos por um fator de segunda ordem; Modelo $4=$ cinco fatores correlacionados; Modelo $5=$ seis fatores com um fator de segunda ordem predizendo os fatores psicológico e socioeconômico; Modelo $6=$ unifatorial.

$* p<0,01$.

O modelo 1 apresenta os melhores indicadores de ajuste em comparação com os modelos 2, 3, 4 e 6 . Já os modelos 1 e 5 , obtiveram índices de ajuste muito próximos. O teste de $\Delta \chi^{2}$ não revelou diferença significativa entre esses dois modelos. Optamos por manter o modelo proposto, já que entendemos que as crenças psicológicas e socioeconômicas são diferentes entre si, e pelo modelo 1 ser mais parcimonioso, já que não pressupõe um fator de segunda ordem. No modelo 1, a inspeção dos IMs (Índices de Modificação) para as saturações (Lambdas, $\lambda$ ) e os erros de medida (Deltas, $\delta$ ) indicou que seria recomendável $(\mathrm{IM}=$ 67,34 ) correlacionar erros de medida dos itens 2 (A pessoa pode ficar doente mental se estudar muito) e 11 (A doença mental tem como causa o estudo em demasia). Procedendo desta forma, a estrutura fatorial resultante permitiu obter melhores indicadores de ajuste aos dados para o modelo proposto: $\chi^{2}(389)=722,25, \mathrm{p}<0,05, \chi^{2} / g l=1,86$, GFI $=$ $0,84, \mathrm{AGFI}=0,81, \mathrm{CFI}=0,89, \mathrm{RMSEA}=0,058(\mathrm{IC} 90 \%$ $=0,052-0,065)$ e ECVI $=3,46$. Neste caso, todos os $\lambda$ foram superiores a 0,30 , sendo estatisticamente diferentes de zero $(\lambda \neq 0 ; z>1,96, p<0,05)$. Na Figura 1 é apresentada a estrutura fatorial deste modelo.

Os índices de consistência interna, alfa de Cronbach $(\alpha)$ e homogeneidade (correlação média interitens, $r_{\mathrm{m} . \mathrm{i}}$ ), foram computados para cada fator, sendo observados os seguintes valores: Psicológicas $\left(\alpha=0,75, r_{\mathrm{m} . \mathrm{i}}=0,38\right)$, Religiosas $\left(\alpha=0,84, r_{\mathrm{m} . \mathrm{i}}=0,52\right)$, Biológicas $(\alpha=0,72$, $\left.r_{\mathrm{m} . \mathrm{i}}=0,34\right)$, Uso de Drogas $\left(\alpha=0,81, r_{\mathrm{m} . \mathrm{i}}=0,45\right)$, Socioeconômicas $\left(\alpha=0,79, r_{\mathrm{mi}}=0,43\right)$ e Contingenciais $(\alpha=$ $\left.0,80, r_{\mathrm{m} . \mathrm{i}}=0,44\right)$. 


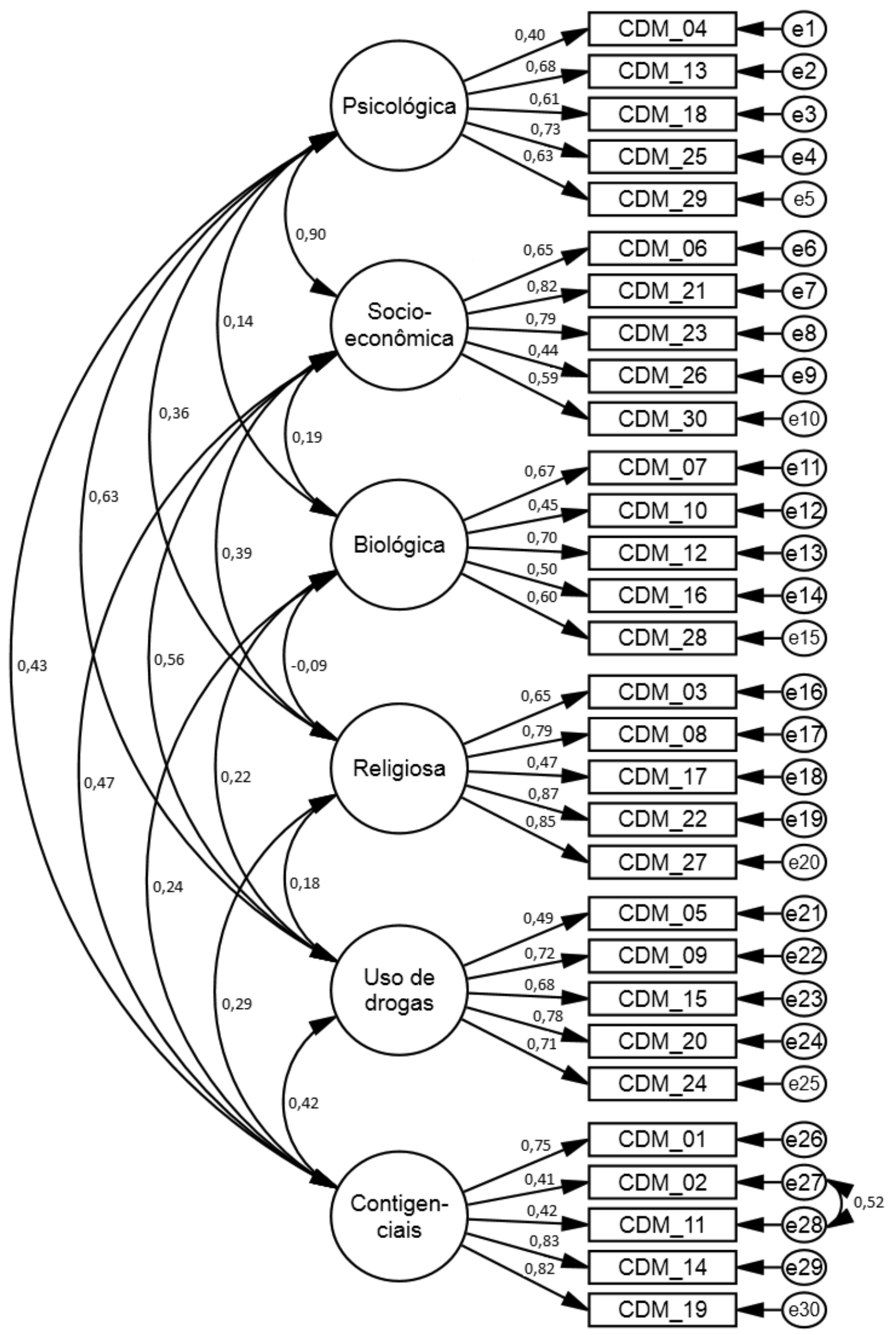

Figura 1. Estrutura fatorial do modelo com seis fatores e suas saturações. 
Maciel, S. C., Pereira, C. R., Lima, T. J. S. \& Souza, L. E. C. (2015). Desenvolvimento e Validação da Escala de Crenças sobre a Doença Mental.

\section{Discussão}

Este terceiro estudo mostrou evidência empírica adicional de validade fatorial e consistência interna da Escala de Crenças sobre a Doença Mental (ECDM). A análise fatorial confirmatória da medida apontou a adequação da estrutura fatorial proposta, embora alguns índices de ajuste obtidos sejam limítrofes ao que é comumente referido na literatura como desejável (Garson, 2012). De maior importância, o teste de modelos explicativos alternativos apontou que o modelo 5, com um fator de segunda ordem predizendo as crenças psicológicas e socioeconômicas, obteve ajuste similar ao do modelo proposto. Não obstante, optamos por manter o modelo proposto, pois as crenças psicológicas e socioeconômicas são conceptualmente diferentes entre si (Minayo, 1988). Enquanto a psicológica está relacionada com fatores de ordem emocional, de estresse e traumas, a socioeconômica diz respeito basicamente à crença de que as condições econômicas escassas levam a doença mental.

Em face de algumas limitações apontadas no Estudo 2, como a quantidade desigual de itens por fator e baixos valores de alfa de Cronbach, no presente estudo novos itens foram elaborados, de maneira que cada fator da escala fosse composto por cinco itens. Todos os fatores apresentaram índices de consistência interna adequados. Em síntese, a estrutura hexafatorial da ECDM foi replicada, assegurando evidências satisfatórias de validade fatorial e consistência interna.

\section{Discussão Geral}

Os estudos apresentados mostram evidências satisfatórias de validade fatorial e consistência interna de uma nova medida acerca das crenças sobre a natureza da doença mental. A ECDM foi construída a partir de entrevistas com profissionais da saúde, familiares de doentes mentais e estudantes universitários. Neste sentido, procurou-se compor uma medida que representa não apenas o saber científico sobre o tema, mas sim as crenças compartilhadas socialmente, por isso procuramos compor a amostra do Estudo 1 com participantes de diferentes extratos sociais e distintos níveis de escolarização. A ECDM busca, também, estruturar o conhecimento sobre as crenças causais da doença mental, contribuindo para uma sistematização das crenças sobre este tema que se encontram disseminadas em diversos estudos (Link et al., 1999; Loureiro et al., 2008; Minayo, 1988; Spadini \& Souza, 2006). Por fim, a ECDM vem preencher uma lacuna existente de medidas sobre as crenças acerca da doença mental, uma vez que alguns instrumentos validados no Brasil e que se aproximam desta temática têm por foco principal avaliar as atitudes (Gonçalves et al., 2008) e as opiniões sobre a doença mental (Pedrão et al., 2005), mas não as crenças específicas sobre a natureza da doença mental, as quais estão na base das atitudes que podem ser medidas por aqueles instrumentos.

Ademais, optou-se por desenvolver uma escala uma vez que aquelas desenvolvidas em outros países para avaliar especificamente as crenças, apresentaram evidências insuficientes de validade e precisão, tais como baixa consistência interna (Lincoln et al., 2008), ou falta de evidências de validade de construto e consistência interna (Link et al., 1999; Loureiro et al., 2008). Não obstante, a ECDM compreende crenças que são comumente descritas nestas escalas, tais como as crenças biológicas e psicológicas (Haqanee et al., 2014; Lincoln et al., 2008; Link et al., 1999; Loureiro et al., 2008; Schlier et al., 2014; Spadini \& Souza, 2006), e também as religiosas (Link et al., 1999). Estas similaridades permitem que sejam feitas comparações com outros estudos, já que a ECDM engloba as crenças com significados similares àquelas que têm sido mais reportadas na literatura.

A escala final, composta por 30 itens, apresenta evidências satisfatórias de validade de construto e de consistência interna, demonstradas em dois estudos, e pode ser empregada para avaliar os tipos de crenças que as pessoas acreditam ser a causa da doença mental. A solução fatorial com seis tipos de crenças se mostrou a mais adequada em ambos os estudos.

Embora a ECDM se apresente como uma opção adequada para mensurar as crenças acerca da doença mental, é possível elencar algumas limitações nos estudos realizados. Particularmente, é necessário assinalar que as amostras não podem ser consideradas como representativas da população brasileira, pois foram constituídas exclusivamente por estudantes universitários. Todavia, cabe ressaltar que as entrevistas que foram empregadas para a construção dos itens foram feitas com amostras de posições socioeconômica e educacional diversificadas. Em termos de estudos futuros, será necessário replicar a ECDM com amostras da população geral. Ademais, a ECDM pode ser empregada em estudos correlacionais e experimentais, juntamente com medidas atitudinais e comportamentais em relação aos doentes mentais, pois as crenças medidas pela ECDM podem ser utilizadas como preditoras do preconceito e da exclusão do doente mental.

\section{Referências}

Angermeyer, M. C., Holzinger, A., Carta, M. G., \& Schomerus, G. (2011). Biogenetic explanations and public acceptance of mental illness: Systematic review of population studies. The British Journal of Psychiatry, 199, 367-372. doi:10.1192/ bjp.bp. 110.085563

Bardin, L. (1977). Análise de conteúdo. São Paulo, SP: Martins Fontes.

Clark, L. A., \& Watson, D. (1995). Constructing validity: Basic issues in objective scale development. Psychological Assessment, 7, 309-319.

Corrigan, P., Markowitz, F., Watson, A., Rowan, D., \& Kubiak, M. (2003). An attribution model of public discrimination towards persons with mental illness. Journal of Health and Social Behavior, 44, 162-179.

Freud, S. (1996). Estudos sobre a histeria. In S. Freud, Estudos sobre a histeria. Rio de Janeiro, RJ: Imago. (Original publicado em 1895) 
Garson, G. D. (2012). Structural Equation Modeling. Asheboro, NC: Statistical.

Gonçalves, S., Abelha, L., Legay, L., \& Lovisi, G. (2008). Equivalência conceitual e semântica da versão em português da Escala Community Attitudes Toward Mentally III (CAMI). Cadernos de Saúde Coletiva, 16, 749-764.

Gureje, O., Olley, B. O., Oluwanuga, O. E., \& Kola, L. (2006). Do beliefs about causation influence attitudes to mental illness? World Psychiatry, 5, 104-107. doi:10.1192/bjp.186.4.331

Haslam, N. (2011). Genetic essentialism, neuroessentialism, and sitgma. Psychological Bulletin, 137, 819-824. doi:10.1037/ a0022386

Haqanee, Z., Lou, E., \& Lalonde, R. N. (2014). Natural kind and entitative beliefs in relation to prejudice toward mental disorders. Journal of Applied Social Psychology, 44, 145-153. doi:10.1111/jasp.12249

Horn, J. L. (1965). A rationale and test for the number of factors in factor analysis. Psychometrika, 30, 179-185.

Lacerda, M., Pereira, C., \& Camino, L. (2002). Um estudo sobre as formas de preconceito contra homossexuais na perspectiva das representações sociais. Psicologia: Reflexão e Crítica, 15, 165-178. doi:10.1590/S0102-79722002000100018

Lincoln, T. M., Arens, E., Berger, C., \& Rief, W. (2008). Can antistigma campaigns be improved? A test of the impact of biogenetic vs psychosocial causal explanations on implicit and explicit attitudes to schizophrenia. Schizophrenia Bulletin, 34, 984-994. doi:10.1093/schbul/sbm131

Link, B. G., Phelan, J. C., Bresnaham, M., Stueve, A., \& Pescosolido, B. A. (1999). Public conceptions of mental illness: Labels, causes, dangerousness, and social distance. American Journal of Public Health, 89, 1328-1333. doi:10.2105/ AJPH.89.9.1328

Loureiro, L. M., Dias, C. A., \& Aragão, R. O. (2008). Crenças e atitudes acerca das doenças e dos doentes mentais. Contributos para o estudo das representações sociais da loucura. Revista Referência, 8, 33-44.

Maciel, S. C., Barros, D. R., Camino, L. F., \& Melo, J. R. F. (2011). Representações sociais de familiares acerca da loucura e do hospital psiquiátrico. Temas em Psicologia, 19, 193-204.

Maciel, S. C., Barros, D. R., Silva, A. O., \& Camino, L. (2009). Reforma psiquiátrica e inclusão social: Um estudo com familiares de doentes mentais. Psicologia: Ciência e Profissão, 29, 436-447.

Matsunaga, M. (2010). How to factor-analyze your data right: Do's, don't's, and how-to's. International Journal of Psychology Research, 3, 97-110.

Minayo, M. C. S. (1988). Saúde-doença: Uma concepção popular da etiologia. Cadernos de Saúde Pública, 4, 363-381.

Morant, N. (2006). Social representations and professional knowledge: The representation of mental illness among mental health practitioners. British Journal of Social Psychology, 45, 817-838. doi:10.1348/014466605X81036

Ngokwey, N. (1988). Pluralistic etiological systems in their social context: A Brazilian case study. Social Science and Medicine, 26, 793-802.

Nunnally, J. C. (1991). Teoría psicométrica. México, DF: Trillas. Oliveira, R. M., Lima, A. E. S., Silva, D. G., Oliveira, M. D. F., \& Alves, K. S. M. (2011). Visão dos acadêmicos de enfermagem em relação ao cliente portador de transtorno mental. Revista Cadernos de Ciência e Saúde, 1, 75-85.

Osinaga, V. L. M., Furegato, A. R. F., \& Santos, J. L. F. (2004). Redução das questões de uma escala de medida de opiniões. Revista Brasileira de Enfermagem, 57, 703-705.
Pasquali, L., Nogueira, A. M., Martins, A. E., \& Martins, F. M. (1987). Atitudes frente ao doente mental. Um modelo etiológico e de medida de atitudes. Revista de Psicologia (Fortaleza), 5, 37-67.

Pedrão, L., Galera, S., Silva, M., Gonzalez, A., Costa, M., Mello e Souza, M. C. B. \& Senmache, G. U. (2005). Perfil das atitudes de formandos em enfermagem frente aos transtornos mentais no Brasil, Chile e Peru. Revista Latino-Americana de Enfermagem, 13, 339-343. doi:10.1590/ S0104-11692005000300008

Pereira, C., Torres, A. R., Falcão, L., \& Pereira, A. (2013). O papel de representações sociais sobre a natureza da homossexualidade na oposição ao casamento civil e à adoção por famílias homoafetivas. Psicologia: Teoria e Pesquisa, 29, 79-89.

Pessotti, I. (2001). A loucura e as épocas (2. ed.). São Paulo, SP: Editora 34.

Pescosolido, B. A., Martin, J. K., Long, J. S., Medina, T. R., Phelan, J. C., \& Link, B. G. (2010). A disease like any other? A decade of change in public reactions to schizophrenia, depression, and alcohol dependence. American Journal of Psychiatry, 16, 1321-1330. doi:10.1176/appi.ajp.2010.09121743

Phelan, J. C. (2002). Genetic bases of mental illness - A cure for stigma? Trends in Neurosciences, 25, 430-431. doi:10.1016/ S0166-2236(02)02209-9

Read, J., Haslam, N., Sayce, L., \& Davies, E. (2006). Prejudice and schizophrenia: A review of the "mental illness is an illness like any other" approach. Acta Psychiatrica Scandinavica, 114, 303-318. doi:10.1111/j.1600-0447.2006.00824.x

Rodrigues, C. R., \& Figueiredo, M. A. (2003). Concepções sobre a doença mental em profissionais, usuários e seus familiares. Estudos de Psicologia (Natal), 8, 117-125. doi:10.1590/ S1413-294X2003000100013

Schlier, B., Schmick, S., \& Lincoln, T. M. (2014). No matter of etiology: Biogenetic, psychosocial and vulnerability-stress causal explanations fail to improve attitudes towards schizophrenia. Psychiatry Research, 215, 753-759. doi:10.1016/j. psychres.2013.12.056

Spadini, L. S., \& Souza, M. C. B. M. (2006). A doença mental sob o olhar de pacientes e familiares. Revista da Escola de Enfermagem da USP, 40, 123-127.

Taylor, S. M., \& Dear, M. J. (1981). Scaling community attitudes toward the mentally Ill. Schizophrenia Bulletin, 7, 225-240.

Tsu, T. M., \& Tofolo, V. (1990). Concepções etiológicas de pacientes psiquiátricas sobre a doença mental. Psicologia-USP, 1, 155-166.

Wetzel, C., Kantorski, L. P., Olschowsky, A., Schneider, J. F., \& Camatta, M. W. (2011). Dimensões do objeto de trabalho em um Centro de Atenção Psicossocial. Revista Ciência e Saúde Coletiva, 16, 2133-2143. 
Maciel, S. C., Pereira, C. R., Lima, T. J. S. \& Souza, L. E. C. (2015). Desenvolvimento e Validação da Escala de Crenças sobre a Doença Mental.

\section{ANEXO A \\ Escala de Crenças sobre a Doença Mental (ECDM)}

Instruções: Abaixo apresentamos uma lista de frases sobre a DOENÇA MENTAL. Gostaríamos que indicasse em que medida concorda com estas frases. Circule o número que melhor representa a sua opinião, de modo que quanto maior for o número circulado, mais forte será a sua concordância com a frase.

1. A doença mental pode ser causada por um traumatismo craniano (queda que machuca a cabeça)

2. A pessoa pode ficar doente mental se estudar muito

3. A doença mental está relacionada com a falta de fé em Deus

4. A doença mental é causada por traumas psicológicos ocorridos na infância

5. Dependentes químicos são mais vulneráveis a adquirir transtornos mentais

6. A doença mental pode ser causada por problemas financeiros

7. A doença mental está relacionada com problemas orgânicos no cérebro

8. A doença mental pode ser uma das manifestações dos espíritos

9. O consumo de drogas causam doenças mentais graves

10. Distúrbios cerebrais estão relacionados com o aparecimento da doença mental

11. A doença mental tem como causa estudo em demasia

12. A doença mental é causada por problemas biológicos

13. A doença mental está relacionada com o excesso de preocupação com a vida

14. Pancadas na cabeça causam doença mental

15. A ingestão de bebidas alcoólicas em demasia favorece o surgimento das doenças mentais

16. A doença mental é determinada por fatores genéticos

17. A doença mental ocorre por vontade de Deus

18. A doença mental é causada por instabilidade emocional

19. Acidentes que atingem a cabeça podem desencadear doença mental

20. Uso de drogas pode causar a doença mental

21. O desemprego contribui para o desenvolvimento da doença mental

22. A doença mental é a expressão de um espírito que entra na mente da pessoa

23. A falta de condições econômicas adequadas é uma das causas da doença mental

24. A doença mental está relacionada com o uso de drogas

25. Uma das causas da doença mental é o excesso de tristeza

26. A falta de alimentação quando criança é uma das causas da doença mental

27. A doença mental está relacionada com a fraqueza para resistir às manifestações dos espíritos

28. A doença mental é um distúrbio orgânico

29. O aparecimento da doença mental é favorecido por problemas emocionais

30. A pobreza é um dos determinantes da doença mental 